\title{
Cryptic plasmids of alfalfa root nodule bacteria - structural and functional diversity
}

\author{
Alla Saksaganskaia \\ ARRIAM, Saint-Petersburg, Pushkin, \\ Russia \\ allasaksaganskaya@mail.ru
}

\author{
Viktoria Muntyan \\ ARRIAM, Saint-Petersburg, Pushkin, \\ Russia \\ vucovar@yandex.ru \\ Marina Roumiantseva \\ ARRIAM, Saint-Petersburg, Pushkin, \\ Russia \\ mroumiantseva@yandex.ru
}

\author{
Alexey Afonin \\ ARRIAM, Saint-Petersburg, Pushkin, \\ Russia \\ afoninalexeym@gmail.com
}

\begin{abstract}
Abundance of plasmids in genomes of root nodule nitrogen fixing bacteria (rhizobia) is their common feature. Plasmids harboring genes related to symbiosis are usually called symbiotic plasmids, while rhizobia can contain differing number of cryptic plasmids, which sizes are varied from $7 \mathrm{kbp}$ to $600 \mathrm{kbp}$. Cryptic plasmids were predicted to be important both for the existence of rhizobia in soil and for formation of effective nitrogen-fixing symbiosis with particular host plant. Nevertheless, assemble and annotation of such replicons became possible only in the postgenomic era. In this work a structural and functional analysis of cryptic plasmids of Sinorhizobium meliloti which forming symbiosis with alfalfa was done. Native isolates of $S$. meliloti were recovered from nodules of alfalfa plants adapted to salinized soils at the Aral Sea region. The sequence analysis was done for four plasmids ranging in size from $31.2 \mathrm{kbp}$ to $453.7 \mathrm{kbp}$. A large number of ORFs of cryptic plasmids of $S$. meliloti are associated with metabolic processes as it was established. For the first time prophage sequences were identified in cryptic plasmids. Data proving that cryptic plasmids of native strains are related to horizontal gene transfer and could be essential for rhizobia fitness.
\end{abstract}

Keywords - S. meliloti, cryptic plasmids, ORFs, COGs, prophages, horizontal gene transfer

\section{Motivation and aim}

\section{Motivation}

Cryptic plasmids (CPs) are accessory elements of the multicomponent genome of $\alpha$-proteobacteria forming nitrogen-fixing symbiosis with legumes [1]. The study of the functional role of nonsymbiotic CPs has got a significant prospect in postgenomic era.

Aim

The aim of this work was the comparative analysis of CPs which were obtained as a result of genome-wide sequencing of symbiotically active strains Sinorhizobium meliloti AK555 and AK83 adapted to salinized area of the Aral Sea region.

\section{Methods}

Strains Sinorhizobium meliloti AK555 and AK83 were obtained in the frame of the INCO-COPERNICUS project (ICA2-CT-2001-10001). Cryptic plasmids assembly of AK555 was done on the basis of full-genome sequencing [2]. The annotation of plasmid sequences was done by using bioinformatics approaches (Flye, Racon, Medaka, Pilon, Prokka).

Data about pSINME02 and pSINME01 of the AK83 are from GenBank (NC_015592.1; NC_015597.1).

\section{Results}

The two nonsymbiotic plasmids as a result of genomewide sequencing of the AK555 were assembled and annotated by using bioinformatics approaches (Flye, Racon, Medaka, Pilon, Prokka). The SMd is a replicon of $31.2 \mathrm{kbp}$ with 34 ORFs and SMe is a replicon of $453.7 \mathrm{kbp}$ containing 519 ORFs. Plasmids of the strain AK83 are: pSINME02 of 70.5 $\mathrm{kbp}$ and pSINME01 of $256.3 \mathrm{kbp}$. The first replicon contained 85 ORFs and the second one 287 ORFs.

The analysis of SMd of the AK555 revealed that $86 \%$ of its length showed $92 \%$ homology with nearly a half of the sequence pSINME02 of the AK83. But no homology was detected between above mentioned plasmids and other two plasmids SMe and pSINME01 of the both strains.

More than a half of ORFs of each examined replicons were characterized by COGs. In the result, all ORFs were allocated into 4 functional groups: cellular processes and signaling (D, $\mathrm{M}, \mathrm{N}, \mathrm{O}, \mathrm{T}, \mathrm{U}, \mathrm{V}, \mathrm{W})$, information storage and processing (J, $\mathrm{K}, \mathrm{L})$, metabolism (C, E, J, H, I, P, Q) and poorly characterized $(\mathrm{R}, \mathrm{S}, \mathrm{X})$. An average portions of ORFs belonged to cellular processes/signaling or metabolism groups were 0.2 and 0.34 , correspondingly, per replicon. ORFs related to metabolism were detected on SMe, pSINME02, pSINME01, but that was not a case for the SMd. On the SMe was detected ORF encoding Kup2 protein involving in $\mathrm{K}+$ transport, which could relate to osmoadaptation process. The Kup2 is homologous to Kup proteins encoded by other two sequences localized on the chromosome and megaplasmid SMa of the same strain. While the homology was 71 and $84 \%$, respectively.

On plasmids SMe and SINME01 prophage sequences of different sizes were detected by using PHASTER (https://phaster.ca/). The 2 sequences of the SMe were: 12.9 $\mathrm{kbp}$ and $5.9 \mathrm{kbp}$, which were characterized as intact and incomplete prophages, correspondingly. On plasmid pSINME01 the 2 prophage sequences of a sizes $43.9 \mathrm{kbp}$ and $9.3 \mathrm{kbp}$ were identified. These sequences were characterized as intact prophage and incomplete prophages. The analysis of protein-coding genes of phage related sequences revealed homology with sequences of phages from Siphoviridae, Myoviridae and Podoviridae families.

Summarizing, cryptic plasmids of $S$. meliloti are enriched with ORFs related to vital metabolic processes, harboured phage related sequences. The fact of the homology between two plasmids of genetically unrelated strains evident that these plasmids are participating in horizontal gene transfer. Thus nonsymbiotic plasmids of $S$. meliloti are essential elements of rhizobia fitness. 


\section{ACKNOWLEDGMENT}

Supported by the RFBR 18-04-01278A (functional role of ORFs) and by RSF 20-16-00105.

\section{REFERENCES}

[1] Lagares A., Sanjuán J., Pistorio M. (2014) The plasmid mobilome of the model plant-symbiont Sinorhizobium meliloti: coming up with new questions and answers. Microbiology Spectrum. 2(5):PLAS-00052013. doi:10.1128/microbiolspec.PLAS-0005-2013.

[2] Muntyan V.S., Baturina O.A., Afonin A.M., Cherkasova M.E., Laktionov Y.V., Saksaganskaya A.S., Kabilov M.R., Roumiantseva M.L. (2019) Draft genome sequence of Sinorhizobium meliloti AK555. Microbiology Resource Announcements. 8(2): e01567-18. doi: 10.1128/MRA.01567-18. 